\title{
Mixed covering of trees and the augmentation problem with odd diameter constraints
}

\author{
Victor Chepoi Bertrand Estellon Karim Nouioua \\ Yann Vaxès ${ }^{1}$ \\ LIF - Faculté des Sciences de Luminy \\ Universitée de la Méditerranée \\ Marseille, France
}

\begin{abstract}
In this talk, we will outline a polynomial time algorithm for solving the problem of partial covering of trees with $n_{1}$ balls of radius $R_{1}$ and $n_{2}$ balls of radius $R_{2}\left(R_{1}<\right.$ $R_{2}$ ) so as to maximize the total number of covered vertices. We will then show that the solutions provided by this algorithm in the particular case $R_{1}=R-1, R_{2}=R$ can be used to obtain for any integer $\delta>0$ a factor $\left(2+\frac{1}{\delta}\right)$ approximation algorithm for solving the following augmentation problem with odd diameter constraints $D=$ $2 R+1$ : given a tree $T$, add a minimum number of new edges such that the augmented graph has diameter $\leq D$. The previous approximation algorithm of Ishii, Yamamoto, and Nagamochi (2003) has factor 8.
\end{abstract}

Keywords: Partial Covering, Diameter, Augmentation problem, Dynamical programming, Approximation algorithms.

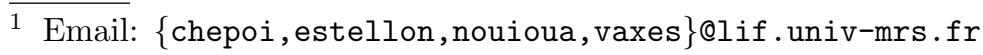


This talk will be divided into two parts. In the first part, we describe a dynamic programming algorithm for solving the following problem :

Problem Partial Mixed Covering: Given a tree $T=(V, E)$ with $n$ vertices, the non-negative integers $R_{1}, R_{2}\left(R_{1}<R_{2}\right)$ and $n_{1}, n_{2}$, locate $n_{1}$ balls of radius $R_{1}$ and $n_{2}$ balls of radius $R_{2}$ so as to maximize the total number of covered vertices.

This problem generalizes the Maximum Coverage problem investigated by Megiddo, Zemel, and Hakimi [6], in which, given a tree $T$ and the integers $R_{0}$ and $n_{0}$, one wish to locate $n_{0}$ balls of radius $R_{0}$ so as to maximize the total number of covered vertices. Our algorithm follows the main lines of the algorithm from [6] and works in general in the following way. Root the tree $T$ at an arbitrary vertex $u$. The algorithm proceeds this tree in a upward manner, from leaves to the root, by solving larger and larger subproblems of the following type. Given the current vertex $s$, and the integers $0 \leq n_{1}^{\prime} \leq$ $n_{1}, 0 \leq n_{2}^{\prime} \leq n_{2}$, the algorithm finds the maximal number of covered vertices of $T_{s}$ in a partial covering using $n_{1}^{\prime}$ balls of radius $R_{1}$ and $n_{2}^{\prime}$ balls of radius $R_{2}$ located in $T_{s}$. However, the algorithm must take care of two things: (i) some ball which will be located outside $T_{s}$ at some later stage and whose radius and center are yet unknown may have an impact on the covering of $T_{s}$, and (ii) we have to consider the interaction between the subtrees rooted at the neighbors of $s$, because some vertices of one or several such subtrees may be covered by a ball located in another subtree. To overcome these difficulties, we introduce two additional parameters $r$ and $a$ which take integer values in the ranges $\left[-1, R_{2}-1\right]$ and $\left[0, R_{2}\right]$, respectively. For fixed values of $r$ and $a$, the algorithm returns the maximal number of covered vertices of $T_{s}$ in a partial covering using $n_{1}^{\prime}$ balls of radius $R_{1}$ and $n_{2}^{\prime}$ balls of radius $R_{2}$ located in $T_{s}$ (permanent balls), given that one additional (temporary) ball of radius $r$ is located at $s$ and that at least one of the permanent balls located in $T_{s}$ covers every vertices outside $T_{s}$ at distance at most $a$ from $s$. This requires the solution of a resource allocation problem, which optimally distributes the balls of radius $R_{1}$ and the balls of radius $R_{2}$ among the subtrees rooted at the neighbors of $s$ in $T_{s}$, using for this the optimal solutions of the previously solved subproblems at each of the sons of $s$.

Notice also that running the algorithm for Partial Mixed Covering for all feasible pairs $\left(n_{1}^{\prime}, n_{2}^{\prime}\right)$, we obtain a polynomial time algorithm for the following problem: 
Problem Mixed Covering: Given a tree $T=(V, E)$ with $n$ vertices, a function $f$ of two non-negative integer variables, the non-negative integers $R_{1}, R_{2}$ $\left(R_{1}<R_{2}\right)$ and $n_{1}, n_{2}$, find a covering (if it exists) of $T$ with $n_{1}^{\prime} \leq n_{1}$ balls of radius $R_{1}$ and $n_{2}^{\prime} \leq n_{2}$ balls of radius $R_{2}$ minimizing the function $f\left(n_{1}^{\prime}, n_{2}^{\prime}\right)$.

In the second part of our talk, we use this polynomial time algorithm to derive an approximation algorithm for the following augmentation problem:

Problem ADC (Augmentation under Diameter Constraints): Given a graph $G=(V, E)$ with $n$ vertices and a positive integer $D$, add a minimum number OPT of new edges $E^{\prime}$ such that the augmented graph $G^{\prime}=\left(V, E \cup E^{\prime}\right)$ has diameter at most $D$.

Due to its practical importance for improving the reliability of existing communication networks, the Augmentation under Diameter Constraints problem has received much attention in the literature $[1,3,4,5,7]$. In particular, it was shown to be NP-hard for any $D \geq 2$ and at least as difficult to approximate as SET COVER $[1,5,7]$.

For the problem ADC on trees, Chepoi and Vaxès [1] presented a factor 2 approximation algorithm for even $D=2 R$ and Ishii, Yamamoto, and Nagamochi [4] presented a factor 8 approximation algorithm for odd $D=2 R+1$. The algorithm in [1] for $D=2 R$ computes an optimal covering of the tree $T$ with one ball of radius $R$ and a minimum number of balls of radius $R-1$ and then, it adds edges between the center of the radius $R$ ball and the centers of radius $R-1$ balls in the covering. For the case $D=2 R+1$ we consider the following feasible augmentation: take a mixed covering of $T$ with $n_{1}$ balls of radius $R-1$ and $n_{2}$ balls of radius $R$ minimizing the function $f\left(n_{1}, n_{2}\right)=n_{1}+\frac{n_{2}\left(n_{2}-1\right)}{2}$ (in this case, Mixed Covering can be solved in time $\left.O\left(n^{3.5} R^{2}\right)\right)$ and draw an edge between any pairs of centers of balls of radius $R$ and between the centers of any balls of radius $R-1$ and the center of some ball of radius $R$. So, in case $D=2 R$ the added edges form a star while in case $D=2 R+1$ they constitute a clique of size $n_{2}$ plus $n_{1}$ pendant edges connected to this clique. In this talk based on the paper [2], we will outline the proof that the algorithm for odd $D$ provides a feasible solution containing at most $\left(2+\frac{1}{\delta}\right) O P T+O\left(\delta^{5}\right)$ added edges for any $\delta>0$, thus asymptotically matching the approximation ratio for even $D$. We conjecture that this algorithm for odd $D$ as well as the algorithm in [1] for even $D$ are optimal up to an additive constant error term. 


\section{References}

[1] V. Chepoi, Y. Vaxès, Augmenting trees to meet biconnectivity and diameter constraints, Algorithmica, 33 (2002), 243-262.

[2] V. Chepoi, B. Estellon, K. Nouioua, Y. Vaxès, Mixed covering of trees and the augmentation problem with odd diameter constraints, submitted to Algorithmica, 2004.

[3] Y. Dodis, S. Khanna, Designing networks with bounded pairwise distance, Annual ACM Symposium on the Theory of Computing, (1999), 750-759.

[4] T. Ishii, S. Yamamoto, H. Nagamochi, Augmenting forests to meet odd diameter requirements, International Symposium on Algorithms and Computation (ISAAC'03), Lecture Notes in Computer Science, 2906 (2003), pp. 434-443.

[5] Ch.-L. Li, S.Th. McCormick, D. Simchi-Levi, On the minimum-cardinalitybounded-diameter and the bounded-cardinality-minimum-diameter edge addition problems, Operations Research Letters, 11 (1992), 303-308.

[6] N. Megiddo, E. Zemel, S.L. Hakimi, The maximum coverage location problem, SIAM J. Alg. Disc. Meth., 4 (1983), 253-261.

[7] A.A. Schoone, H.L. Bodlaender, J. van Leeuwen, Diameter increase caused by edge deletion, J. Graph Theory 11 (1987), 409-427. 